\title{
A New Mass Spectrometer
}

By W. R. Smythe and J. Mattauch* California Institute of Technology

(Received March 22, 1932)

\begin{abstract}
A new type of mass spectrometer has been constructed in which no magnetic fields are used. By applying an alternating electric field at right angles to the beam of positive particles all but certain velocities are removed from the beam. Because of the fact that a symmetry not contemplated in the theory previously published was introduced in the construction for mechanical reasons, the apparatus transmits some other velocities besides those desired. This produces extra peaks and complicates the mass spectrum. Because of this and the fact that the apparatus is badly contaminated with mercury from the diffusion pumps in spite of the continued use of liquid air, we have designed a new apparatus in which, it is expected, both effects will be eliminated. Comparison of the peak at 32 with that at 34 gives a maximum ratio of the intensity of $\mathrm{O}_{16} \mathrm{O}_{16}$ to $\mathrm{O}_{16} \mathrm{O}_{18}+\mathrm{Hg}_{204}^{6+}$ of 300 . This is presumably the ratio with the smallest contamination of mercury and means that the ratio of $\mathrm{O}_{16}$ to $\mathrm{O}_{18}$ is greater than 600 .
\end{abstract}

SOME time ago ${ }^{1}$ one of the authors described a method of sorting out, from $S_{\text {a beam of charged particles, those having a certain velocity, regardless }}$ of the mass. The plan was to apply, at right angles to the beam, alternating electric fields in such a way that only those particles having a certain velocity would pass through them undisplaced and undeflected. By expressing the amplitude of the fields as a function of position, in terms of a Fourier's series it was shown that certain symmetry conditions were required of the fields to produce the desired results. An arrangement satisfying these conditions was suggested, in which there are two separate fields, the distance between their centers being such that a particle having the specified velocity enters the second field when the phase is opposite to that in which it entered the first. The fields are alike in form and if we take the origin at the center of one, its amplitude, $f(x)$, must equal $f(x-a)$, where $2 a$ is the length of the field. Particles traveling in the $x$ direction in such a pair of fields with a velocity $v$ will emerge undeviated and undeflected if $v=2 a \nu / n$, where $\nu$ is the frequency of the field and $n$ is an odd integer. It was pointed out that the beam emerging from such a filter could be analyzed for mass by either electric or magnetic deflection. The latter method gives twice the mass resolving power of the first because it is less sensitive to inhomogeneity of velocity.

A mass spectrometer using the above principles has been constructed as shown in Fig. 1. Two similar pairs of condensers are used, the upper plate of each pair being fastened to the same piece of plate glass, which, in turn, is fastened to the top of a brass box with partitions as shown. The lower plates are similarly mounted on the lower side of the box. Each box fits in accurately centered rings in a long brass tube. One box is clamped in position when the apparatus is assembled, but the other can be moved along the axis of the tube when the apparatus is in operation by means of the rack and pinion

* International Research Fellow.

${ }^{1}$ Smythe, Phys. Rev. 28, 1275 (1926). 
shown, and its position observed through a window. Electrical contact is made to the condensers by spring plungers which rest against the strips which connect each condenser pair. Slits placed at $S_{1}$ and $S_{2}$ serve to collimate the beam, and slits at $S_{3}$ and $S_{4}$ exclude that portion which has been displaced or deviated in the fields.

The aluminum cathode shown at the extreme left in Fig. 1 is pierced by a rectangular channel $30 \mathrm{~mm}$ long having a cross section $0.5 \mathrm{~mm}$ by $3 \mathrm{~mm}$. Since both the positive rays and the gas from the discharge tube pass through this channel a pump exit and a second similar channel are placed between the cathode and the first slit at $S_{1}$. This pumping tube is connected between the two stages of a mercury diffusion pump and the other two pumping tubes are connected on the high vacuum side of the pumps. In this way, with a three

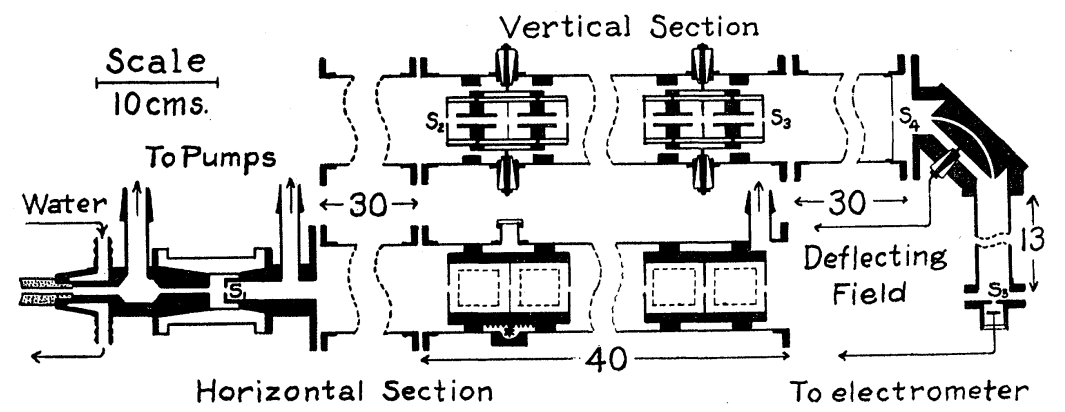

Fig. 1. Schematic diagram of the mass spectrometer.

centimeter dark space in the discharge tube, there seems to be very little scattering of the positive beam in the 1.25 meters between $S_{1}$ and $S_{5}$, when there are no leaks.

At $S_{4}$ the beam, containing only certain velocities, emerges from the filter, is deflected at right angles by an electrostatic field, measured with a Wolff potentiometer, and impinges on slit $S_{5}$. Behind this slit is a plate connected to a Hoffman electrometer. The electrometer current, plotted as a function of the deflecting voltage, shows peaks at certain values which are proportional to the masses of the positive ions present. In measuring currents the electrometer is disconnected from ground and the zero read. Then, by an automatic telechron timing device, the deflecting field is turned on for an integral number of seconds, the interval being accurate to about 0.001 second, and the deflection read after the needle comes to rest. Intervals of one second are used for the strong peaks and of a minute or more for the very weak ones. Since the instrument and shields surrounding the leads are evacuated there is no readable leakage in ten or fifteen minutes. When the positive current is steady none of a group of six measurements, made with the same deflecting field and giving a deflection of $20 \mathrm{~cm}$ or more, should deviate more than one part in 2000 from the mean.

The alternating field applied to the filter is supplied by a crystal-controlled oscillator, containing four UX 210 tubes. The crystal is mounted in a watercooled holder in the grid circuit of the first tube and a slight feedback is neces- 
sary to keep it going. The output from this tube is then applied to the grid of a second tube which is kept so strongly negative with a $B$ battery that no plate current flows unless the crystal is oscillating. In this way current pulses having the crystal frequency $(\lambda=120 \mathrm{~m})$ flow in the plate circuit of the second tube. Two inductively coupled and neutralized circuits, which can be tuned to wave-lengths between $25 \mathrm{~m}$ and $70 \mathrm{~m}$ permit amplification of the second $(60 \mathrm{~m})$, third $(40 \mathrm{~m})$ and fourth $(30 \mathrm{~m})$ harmonics of this pulsating current. The filter condensers form part of a tuned circuit inductively coupled to the last stage of the amplifier. The strength of the alternating field is controlled by the tuning of this last circuit. This tuning and that of the amplifying circuits has no measurable effect on the frequency. This was proved by beating with another circuit in a distant part of the building and tuning the beat note to a flute. No variation in the pitch of this note could be detected when the tuning dials were turned.

The discharge tube consisted of a Pyrex cylinder $20 \mathrm{~cm}$ long and $5 \mathrm{~cm}$ in diameter except around the cathode where it was contracted to $2.5 \mathrm{~cm}$ diameter. It is mounted, with a little graphite to prevent sticking, on the water cooled cone shown at the left of Fig. 1, and beeswax is run around the outside after mounting. A silicon metal anticathode is fixed about $5 \mathrm{~cm}$ from the cathode face in the center of the tube. When the tube is in operation the anticathode usually runs at a bright red heat. The anode is an aluminum cylinder mounted in a side tube $15 \mathrm{~cm}$ from the cathode face. The gas to be analyzed enters the tube through a liquid air trap on the side opposite the anode. The voltage for the discharge tube is the rectified output from a pair of neon sign transformers. The tube is usually run at from 5000 to 8000 volts, as read on a Braun electrostatic voltmeter, and a current of from 10 to 25 milliamperes. At first great difficulty was experienced in holding the discharge steady but this was remedied by installing a stabilizing tube. Between the cathode, which is grounded, and one side of the transformers is inserted a resistance which may be adjusted, in $10,000 \mathrm{ohm}$ steps, from 0 to 100,000 ohms. The negative end of this resistance is connected to the grid of a UX 852 vacuum tube and the positive end to the filament. The negative voltage on the grid is therefore proportional to the current through the discharge tube. The plate is connected to the discharge tube anode. The resistances and tube are immersed in transformer oil. Without the stabilizer and with the gas supply constant, the current and voltage may be steady for half an hour and then gradually the current decreases, the voltage increases and the gas "cleans up." Finally the discharge becomes intermittent. When watching the voltmeter very carefully it is possible to prevent this by dropping the voltage slightly at the proper time. If care is not taken however the reverse process will then occur. With the stabilizer, when the current through the discharge tube begins to drop the grid voltage of the stabilizer also drops, permitting current to flow in the plate circuit in parallel with the discharge tube. This lowers the voltage across the latter and prevents further change. The discharge runs steadily for an indefinite period with this arrangement. Ballast tubes are used in the primary circuit of the transformers supplying the discharge tube. 
The first tests of the apparatus were made with air, the latter being admitted from the room through a leak consisting of a copper wire sealed in Pyrex. The magnitude of the leak depended on the size of the wire and could be further regulated by sending a current through a few turns of resistance wire around the seal, the thermal expansion of the copper wire finally closing the leak. It was found at once that there were a great many more peaks than could be accounted for by the composition of air and to simplify the mass spectrum it was decided to use pure oxygen. At first mercuric oxide and later lead peroxide were placed in a side tube and heated with a furnace. The pressure in the discharge tube could be very precisely controlled by regulating the heating current in this furnace. With the apparatus set to transmit ions passing the condensers in three cycles $(n=3)$, there appeared, in addition to the expected peak at $X$, "ghost" peaks, also due to the oxygen molecule, and
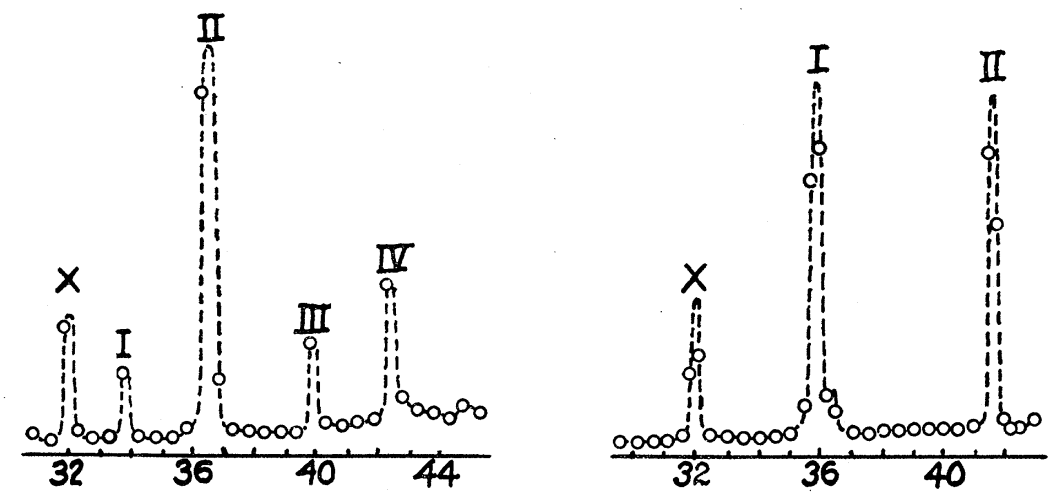

Figs. 2 and 3. Mass spectrometer readings for oxygen. In addition to the expected peak at $\mathrm{X}$ "ghost" peaks appeared at I, II, III, and IV. By moving the condenser pairs over their range of adjustment the position of these ghost peaks varied continuously from that shown in Fig. 2 to that of Fig. 3.

marked I, II, III, IV in Figs. 2 and 3. By moving the condenser pairs over their range of adjustment the position of these ghost peaks varied continuously from that shown in Fig. 2 to that of Fig. 3. For $n=1$ no "ghost" peaks were found and for $n=5$ they were worse. The width of $X$ is about that to be expected for electric analysis for the adjustments used. As previously computed, this width is inversely proportional to $n$. The presence of the extra peaks practically destroys the value of the present apparatus for heavy positives as it prevents the use of the high values of $n$ which give the high resolving power. One way to eliminate these was found. A rough filter, operating on a separate oscillator, was installed between slits $S_{1}$ and $S_{2}$ (Fig. 1). When used at $n=1$ this filter transmitted a broad peak and the frequency could be so adjusted that this coincided with any of the peaks shown in Figs. 2 and 3. This surpressed the other peaks and gave almost no loss in intensity in the velocity transmitted but broadened the base of the peak. This broadening could probably have been eliminated by inserting a collimating section between the two filters. 
The origin of the "ghosts" has been investigated by a reconsideration of the theory which indicates that they are due mainly to the fact that, to simplify the mechanical construction, we made $f(x)$ equal to $f(-x)$. The effect of this was not considered in the original theory but it is not difficult to show that it permits a large additional number of velocities to satisfy the condition for no deflection. If any of these velocities also satisfy the condition for no displacement over any appreciable phase range they will produce "ghosts". The latter condition can not be computed without actually knowing the Fourier coefficients.

A new instrument has been designed, in which it is believed the ghosts will be eliminated completely. Another feature will be the use of butyl phthalate in the diffusion pumps. In the present apparatus, in spite of great care taken to keep liquid air on the traps when evacuated, mercury vapor from the diffusion pumps has contaminated the interior badly, and cannot be removed from the brass and solder of which the apparatus is constructed.

Some of the advantages of this type of mass spectrometer should be noted. The first is the reproducibility of the results. When the oscillator is switched on the peaks are always found at the same value of the deflecting voltage. Another is the ease with which ions of large mass can be compared directly with those of small mass without introducing cumulative errors. For example, if the 60 meter harmonic brings an ion of mass 72 at a certain deflecting field then switching to the 40 meter harmonic will bring mass 32 to exactly the same deflecting field. Since the frequencies of the harmonics of an electric circuit are exact multiples of the fundamental to an extremely high order of precision, this permits the comparison of masses 32 and 72 with the same deflecting field and hence eliminates errors due to potentiometer defects, thermal e.m.f.'s, contact potentials, etc. Another feature is the variable resolving power. By using a weak oscillating field broad and intense peaks are obtained which may be easily located by taking comparatively few points. The peaks may then be narrowed down by increasing the field and studied more accurately.

Some work done last August on the isotope of oxygen of mass eighteen might be mentioned here. We compared the intensity of the peaks at 32 and 34. We found that $\mathrm{Hg}^{6+}$ was invariably present, even when we were preparing our oxygen by decomposing $\mathrm{PbO}_{2}$ and passing it through liquid air and when a visual examination of the optical spectrum showed no mercury lines. The largest value of the ratio of the peaks, corrected for all known errors, was about 300 . In this case we know from the intensity distribution between 32 and 34 that $\mathrm{Hg}^{6+}$ was still present. We may therefore conclude that the ratio of $\mathrm{O}^{16}$ to $\mathrm{O}^{18}$ is greater than 600 . It will be easy to determine the ratio with some precision when we get an apparatus free of mercury.

We are in debt to many people for advice and assistance in constructing this apparatus. In particular we wish to thank Dr. John Pearson for his assistance with the crystal oscillator and Messrs. Pearson, Breslar, and Merkel of the instrument shop for their helpful suggestions and skillful workmanship. 